Kinderkrankenpflege

\title{
Pflege-Assessment für die Pädiatrie
}

- Das neue ePA-Kids2 ist eine konsequente Weiterentwicklung der bewährten Methode "ergebnisorientiertes Pflege-Assessment" (ePA-AC). Damit ist es möglich, die besonderen Anforderungen der Kinderkrankenpflege mit einem durchgängigen Assessmentinstrument abzubilden.

Das ePA-Kids2 ist wie alle anderen Assessments der Methode ePA kompatibel zu anderen Systemen wie Pflegediagnosen oder LEP-Pflegemaßnahmen, so dass der komplette Pflegeprozess einfach und sicher abgebildet werden kann. Damit steht der Kinderkrankenpflege erstmals ein integriertes Instrument zur Verfügung, das sowohl den Pflegefachkräften in der Praxis als auch dem Pflegemanagement Unterstützung im täglichen Handeln und Entscheiden bietet. Das ePA-Kids2 kann von der Geburt bis zum Ende der Behandlung in der Kinderklinik (auch 18 Jahre +) eingesetzt werden. Es bildet die Komplexität des Unterstützungsund Pflegebedarfs (ausgedrückt durch den SelbstStändigkeitsIndex SSI; vergleichbar zum SelbstPflegelndex SPI), die Fähigkeiten der Eltern, das Kind in der aktuellen Situation zu unterstützen (ausgedrückt durch den KompensationsFähigkeitsIndex KFI) und die tatsächliche elterliche Unterstützung (ausgedrückt durch den Übernahmelndex Übl) ab.

Im Pflege-Cockpit sind die wichtigsten pflegerelevanten Informationen zu den Risiken Dehydratation, Mangelernährung/ PYMS, Sturz, Pneumonie, Dekubitus und Abklärungserfordernis Delir/Verwirrtheit auf einen Blick und mit integrierter Berechnung abgebildet. Automatisch werden das Kontinenzprofil (gemäß DNQP) abgeleitet und das Assessment dem Alter des Kindes angepasst. Komplett integriert sind die Gründe für PKMS-J und -K zur vollautomatischen Ausleitung der entsprechenden OPS-Ziffern ohne Doppeldokumentation. Mit Hilfe von Triggerpunkten wird der gesamte Prozess gesteuert. Kennzahlen aus der Routinedokumentation werden ebenfalls generiert.

www.epa-cc.de

\section{Chic und gesund \\ Kompressionsstrümpfe mit Rundumdruck}

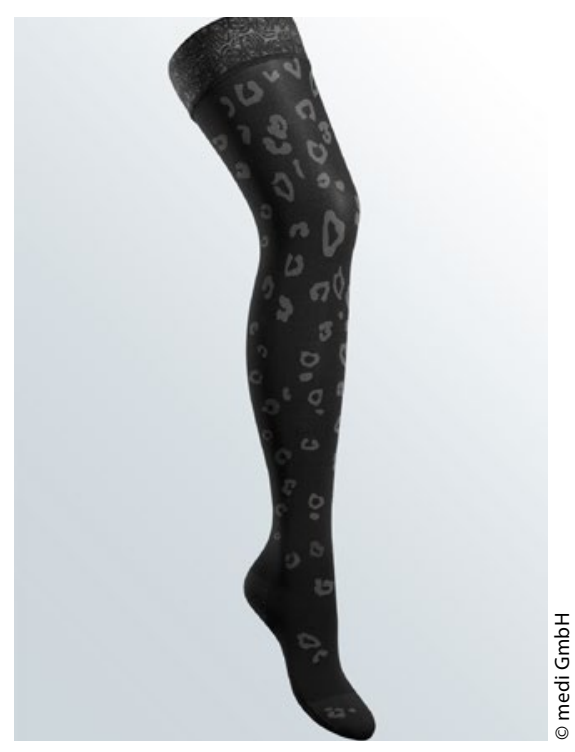

— Der Hersteller medi bringt erstmals rundum bedruckte medizinische Kompressionsstrümpfe auf den Markt. Die Strümpfe in den limitierten Designs "Leo" und "Circle" sind seit Juni 2016 im medizinischen Fachhandel (Sanitätshaus) erhältlich. Beide Designs wurden mit dem Red Dot Award 2016 ausgezeichnet.

Venenleiden sind eine Volkskrankheit. Dazu zählen geschwollene Beine und Füße, Besenreiser oder Krampfadern. Unbehandelt können eine Thrombose oder später ein offenes Bein die Folge sein. Zur attraktiven und bequemen Basistherapie zählen medizinische Kompressionsstrümpfe, beispielsweise mediven elegance von medi. Der definierte Druck verengt die Venen und unterstützt die Muskelpumpen. Das Blut wird besser und schneller zum Herzen gepumpt. Schwellungen klingen ab, die Beine fühlen sich wieder entspannter an. Ein weiterer, schöner Nebeneffekt: Kompressionsstrümpfe modellieren sanft die Beine.

Der Arzt kann medizinische Kompressionsstrümpfe bei Notwendigkeit verordnen. Grundsätzlich übernimmt die Krankenkasse einen Teil der Kosten. Die Strümpfe sind auch freiverkäuflich im medizinischen Fachhandel erhältlich. Dort werden sie individuell angemessen.

www.medi.de 